\title{
Linear radial structure of reactive energetic geodesic acoustic modes
}

\author{
Z.S. Qu ${ }^{1}$, M.J. Hole ${ }^{1}$ and M. Fitzgerald ${ }^{2}$ \\ ${ }^{1}$ Research School of Physics and Engineering, the Australian National \\ University, Canberra ACT 0200, Australia \\ ${ }^{2}$ CCFE Fusion Association, Culham Science Centre, Abingdon, Oxon, OX14 \\ 3DB, UK. \\ E-mail: zhisong.qu@anu.edu.au
}

\begin{abstract}
In this paper we have developed a fluid model to study the radial mode structure of the reactive energetic geodesic acoustic modes (reactive EGAMs), a branch of GAM that becomes unstable in the presence of a cold fast ion beam. We have solved the resulting dispersion relationship, a second order ODE, both analytically in restricted cases and numerically in general. It is found that the reactive EGAM global mode structure is formed with the inclusion of fast ion finite drift orbit effects. In two cases with typical DIII-D parameters but different $q$ profiles, the global EGAM frequency is slightly higher than the local EGAM extremum, located either on axis with a monotonic shear or at mid-radius with a reversed shear. The mode wavelength roughly scales with $L_{\text {orbit }}^{1 / 2}$ in the core and $L_{\text {orbit }}$ at the edge, though the dependency is more complicated for the reversed shear case when $L_{\text {orbit }}<0.06 a\left(L_{\text {orbit }}\right.$ is the fast ion drift orbit width and $a$ the minor radius). Finally, the growth rate of the global mode is boosted by $50 \%$ to $100 \%$ when switching from co-beam to counter-beam, depending on the fast ion density, which may help to explain the more frequent occurrence of EGAMs with counter-injection in experiments.
\end{abstract}

\section{Introduction}

The energetic-particle-induced geodesic acoustic modes (EGAMs) are $n=0$ coherent fluctuations in toroidal magnetic confined plasmas. They were first observed in DIII-D experiments [1] and later in other machines [2, 3]. Their frequencies appear at half of the conventional geodesic acoustic mode (GAM) [4] frequency of the thermal plasma and the beam transit frequency. The EGAMs have caught the interest of the fusion community because they demonstrate a drop of neutron rate during the mode activities that clearly indicates a loss of fast ions [5]. This loss should be minimized in order to achieve a better energy confinement. A suppression of turbulence transport was also observed in accordance with excited EGAMs in a gyrokinetic simulation [6].

The first theory of EGAMs was developed by Fu [7], identifying EGAMs as energetic particle modes (EPMs) whose frequencies and mode structures are nonperturbatively determined by the energetic particles. They were believed to be driven unstable by the fast ions themselves through a positive energy gradient in the distribution function (inverse Landau damping). Follow up theory and simulation studies $[8,9,10,11,12,13,14,15]$ confirmed these findings when the fast ion 
distribution function was taken to be a slowing down distribution or a shiftedMaxwellian. Still, a puzzle remains regarding the turn-on of the mode in $1 \mathrm{~ms}$ right after beam switch-on, a time scale much shorter than the beam collisional slowing down time, indicating an insufficient drive through the wave-particle interaction. This puzzle has been studied by Cao et al [16] and Berk and Zhou [17] separately, giving different explanations. However, it was recently found by Qu et al [18] from a fluid model that the unstable EGAMs could exist, even in the case with a monoenergy, mono-pitch-angle fast ion distribution function. In contrast to the former interpretation, reactive contributions, rather than kinetic (dissipative) contributions, dominate the instabilities. This is in strong analogy to the instabilities in a beamplasma system, in which a cold beam leads to the reactive two-stream instabilities, while the kinetic bump-on-tail instabilities take place when the slope of the fast electron energy distribution function is gentle. Although based on a local theory where the fast ions do not move off the flux surfaces (zero drift orbit width and zero Larmor radius), the theory of reactive EGAMs has helped to solve the puzzle of early turn-on and provided insight to the nature of EGAMs from a fluid perspective.

Nevertheless, as observed experimentally [1], EGAMs are not localized. Rather, their radial wavelength is comparable to the minor radius of the machine. Also, the fast ions drift away from their initial flux surfaces due to the magnetic gradient and curvature drift, making the fast ion finite drift orbit width (FOW) effects important. The radial structure of the kinetically driven EGAMs in DIII-D experiments were reproduced by $\mathrm{Fu}[7]$ using hybrid simulations. Using the small expansion factor $\delta=L_{\text {orbit }} / L_{\text {mode }}$, where $L_{\text {orbit }}$ is the fast ion drift orbit width and $L_{\text {mode }}$ is a measurement of the radial wavelegnth, $\mathrm{Fu}$ has found analytically that the radial wavelegnth is related to the orbit width of the fast particles, as well as the fast ion density and the radial scale length of the thermal GAM frequency. A similar expansion was adapted by Qiu et al [8] in a fully kinetic theory, in which the radial EGAM mode structure was described by a WKB approximation and asymptotic matching, with the continuum damping of the thermal GAM taken into account. With the same expansion factor $\delta$, Zhou [19] studied the global EGAMs driven by either trapped and passing fast ions from a variational principle. All the above analysis were intended for EGAMs driven unstable by wave-particle interaction, while the mode structure of the reactive regime remains unexamined. The purpose of this work is to extend the fluid theory in $\mathrm{Qu}$ et al to resolve the radial mode structure of reactive EGAMs. In this work, we sometimes omit the word "reactive" for simplicity. The wording "EGAM" in this work refers to the reactive EGAM and should not be confused with the wave-particle driven EGAMs in previous works.

We will adapt the same small expansion parameter $\delta=L_{\text {orbit }} / L_{\text {mode }}$, where $L_{\text {orbit }}$ and $L_{\text {mode }}$ are defined by

$$
L_{\text {orbit }} \sim q \rho_{\|}, \quad L_{\text {mode }} \sim\left|\frac{d \ln E_{r}}{d r}\right|^{-1},
$$

with $q$ the safety factor, $\rho_{\|}=m_{i} v_{\|} / e B$ the parallel Larmor radius, $m_{i}$ the ion mass, $e$ the unit charge, $B$ the magnetic field strength, $v_{\|}$the parallel velocity and $E_{r}$ the radial electric field. We have assumed that the fast ions and the thermal ions are of the same type of singly charged ions, i.e. having the same mass $m_{i}$ and charge $e$. Still, the difficulty of fluid closure remains, to truncate the fluid moment hierarchy at the level of pressure. For a local theory where FOW effects are ignored, the CGL theory [20] can give an accurate description of the fast ion response to the field in the high $q$ limit 
$[9,21]$. However, when FOW effects are not negligible, corrections are needed for the CGL double-adiabatic law (see for example [22]). Ignoring finite Larmor radius (FLR) effects and assuming gyro-orbit symmetry, the pressure tensor can still be written in a diagonal form $\overline{\boldsymbol{P}}=p_{\perp}(\overline{\boldsymbol{I}}-\boldsymbol{b} \boldsymbol{b})+p_{\|} \boldsymbol{b} \boldsymbol{b}$, with $p_{\perp}$ the perpendicular pressure, $p_{\|}$ the parallel pressure, $\overline{\boldsymbol{I}}$ the unit dyad, and $\boldsymbol{b}$ the unit vector in the direction of the magnetic field. Due to the smallness of the energy in the drift velocities compared to the energy of the gyro motion $\left(O\left(\epsilon^{2} \delta^{2}\right)\right)$, the value of $p_{\perp}$ can still be approximated by the moment of $\mu B$, where the magnetic moment $\mu$ is given by $\mu=m v_{\perp}^{2} / 2 B$ and is a conserved quantity in the absence of FLR effects. The difficulty of fluid closure is avoided in our current work by limiting the fast ions to have a mono-energy, monopitch-angle distribution function, with which the effective parallel pressure is zero and the perpendicular pressure perturbation is linked to the density perturbation due to the conservation of $\mu$.

In this work, there are three small unitless parameters: the fast particle orbit width over mode width $\delta$, the inverse aspect ratio $\epsilon$ and amplitude of perturbation. All quantities will be equilibrium quantities unless denoted with tilde. Additionally, we consider three species: fast ions, thermal ions and thermal electrons. All quantities will refer to the fast species unless denoted with "e" or "i". Our approach to the problem and structure of work is as follows. Section 2 describes the simplified geometry of the problem: a large aspect ratio, low beta tokamak with concentric flux surfaces. A consistent treatment of the equilibrium fast ion density profile with FOW effects included is also given. In Section 3, we derive the linear fluid theory of reactive EGAMs. We will start with the local theory, reproducing the dispersion relationship in Qu et al. In new work, we keep higher order terms in $\delta$, giving the global dispersion relationship. This dispersion relationship is studied in Section 4, with qualitative analytic discussions and numerical solutions. Dependency of the results on different $q$ profiles is examined. A numerical scan on the relationship between $L_{\text {mode }}$ and the drift orbit width is also performed. In addition, we have found the distinction in mode frequency and growth rate between the counter/co-injection due to the fact that drift orbits of counter(co)-passing ions shift inward (outward) with respect to the flux surfaces, an element omitted in our previous work. The dependency of growth rate with different injection directions agrees with experimental observations. Finally, Section 5 draws the conclusion, and proposes other validation and verification studies.

\section{Equilibrium}

\subsection{Geometry}

In order to obtain a self-consistent tokamak equilibrium with fast ions, we need to solve the anisotropy and flow modified Grad-Shafranov equation $[23,24,25]$ for the equilibrium profiles in radial direction to obtain the poloidal flux function $\Psi(R, Z)$. However in this work, we will ignore the influence of the plasma current and pressure on geometry, except for the existence of a poloidal field. We start with a tokamak plasma with large aspect ratio, circular cross section and low $\beta$. Large asepct ratio means $\epsilon \equiv a / R_{0} \ll 1$, in which $R_{0}$ is the major radius on axis and $a$ the minor radius. We can now use a simplified set of coordinate $(r, \theta, \varphi)$, labeling the radial coordinate, the poloidal and toroidal angle, respectively. The outward shift of the flux surfaces, namely the Shafranov shift, is ignored in our treatment. The local major radius and the magnetic field strength are approximately given by $R \approx R_{0}\left[1+\left(r / R_{0}\right) \cos \theta\right]$ and 
$B \approx B_{0}\left[1-\left(r / R_{0}\right) \cos \theta\right]$. Symmetry in equilibrium is assumed for $\varphi$ direction in a tokamak plasma. For convenience, we define the bi-normal unit vector $\hat{\boldsymbol{\pi}} \equiv \hat{\boldsymbol{b}} \times \hat{\boldsymbol{e}}_{\boldsymbol{r}}$. So we now have another set of orthogonal unit vector triad $\left(\hat{\boldsymbol{e}}_{\boldsymbol{r}}, \hat{\boldsymbol{\pi}}, \hat{\boldsymbol{b}}\right)$. We also have the identity

$$
\nabla \cdot \hat{\boldsymbol{\pi}} \approx-\hat{\boldsymbol{\pi}} \cdot \nabla \ln B \approx(\hat{\boldsymbol{b}} \cdot \nabla \hat{\boldsymbol{\pi}}) \cdot \hat{\boldsymbol{b}}=-\kappa_{g} \approx-\sin \theta / R_{0},
$$

which are all considered as geodesic curvature.

\subsection{Fast ion density profile with finite orbit width}

The fast particle unperturbed guiding centre drift orbits in a tokamak plasma have three constants of motion: the magnetic moment $\mu$, the energy $E$, and the toroidal canonical momentum $P_{\varphi}=-e \Psi+m v_{\|} R$. That is to say, the equilibrium fast ion distribution function can be written as a function of these three quantities only, i.e. $F_{0}=F_{0}\left(E, \mu, P_{\varphi}, \sigma\right)$, where $\sigma$ denotes the type of particle orbit (co/counter passing, trapped). In a toroidal geometry, $B$ is not a constant on flux surfaces. The conservation of $\mu$ will lead to a different perpendicular energy $\mu B$ at different poloidal angle, and thus inhomogeneous fast ion density and pressure on a flux surface, if the distribution of the fast ions is not an unshifted Maxwellian. Moreover, the deviation of constant $P_{\varphi}$ surfaces from constant $\Psi$ surfaces will contribute to this inhomogeneity, since on a constant $\Psi$ surface, the density at different poloidal angle corresponds to the distribution function at different $P_{\varphi}$. The contributions from both effects should be taken into account.

The fast ion distribution function is given by

$$
F_{f}=\frac{m_{i}^{1 / 2} \sqrt{E-\mu B_{0}}}{\sqrt{2} \pi E} n_{f}\left(P_{\varphi}\right) \delta\left(E-E_{0}\right) \delta\left(\Lambda-\Lambda_{0}\right),
$$

where $\Lambda \equiv \mu B_{0} / E$ is the pitch angle and $\delta(x)$ the Dirac delta function. All the fast ions now have the same energy $E_{0}$ and pitch angle $\Lambda_{0}$, consistent with the early beam injection scenario, while the fast ion density at different radial location is described by $n_{f}$. Equation (3) implies that the parallel pressure $p_{\|_{f}}=0$ and the perpendicular pressure $p_{\perp_{f}}=n_{f} E_{0} \Lambda_{0}+O(\epsilon)$.

We now write the fast ion fluid's equilibrium velocity as

$$
\boldsymbol{V}_{f}=V_{f \|} \hat{\boldsymbol{b}}+\boldsymbol{V}_{f, \mathrm{mag}}+\boldsymbol{V}_{f, \mathrm{dia}},
$$

with $V_{f \|}, \boldsymbol{V}_{f, \operatorname{mag}}$ and $\boldsymbol{V}_{f \text {,dia }}$ accounting for the collective transit motion of the fast ions, the magnetic gradient/curvature drift, and the diamagnetic drift, respectively.

Using the ordering in $\delta$, we solve the continuity equation and the momentum equation order by order. We can write the fast ion density $n_{f}$ into $n_{f}=n_{f, \delta^{0}}+n_{f, \delta}+\cdots$ with increasing order in $\delta$. Taking the zeroth order in $\delta$, the equilibrium continuity equation for the fast ions is written as

$$
\nabla \cdot\left(n_{f, \delta^{0}} V_{f \|} \hat{\boldsymbol{b}}\right)=0 .
$$

Equation (5) leads to the well known condition of poloidal flow that $n_{f, \delta^{0}} V_{f} / B$ being a constant on a flux surface. Similarly, we have the lowest order fast ion momentum equation given by

$$
\begin{array}{r}
\nabla \cdot\left[p_{\perp f, \delta^{0}} \overline{\boldsymbol{I}}+\left(m_{f} n_{f, \delta^{0}} V_{f \|}^{2}-p_{\perp f, \delta^{0}}\right) \hat{\boldsymbol{b}} \hat{\boldsymbol{b}}\right]= \\
e n_{f, \delta^{0}}\left[\boldsymbol{V}_{f, \mathrm{mag}}+\boldsymbol{V}_{f, \text { dia }}\right] \times \boldsymbol{B} .
\end{array}
$$


Combining Eq. (5) and the parallel direction of Eq. (6), we will reach that

$$
\left.B \frac{\partial n_{f, \delta^{0}}}{\partial B}\right|_{r}=n_{f, \delta^{0}}\left(1+\frac{1}{2} \frac{\Lambda_{0}}{1-\Lambda_{0}}\right)=n_{f, \delta^{0}}\left(1+c_{f}\right),
$$

showing the change of density on a flux surface due to $\mu$ being an orbit invariant and the distribution function being non-Maxwellian. blackNote that as $\Lambda_{0}$ approaches 1 , the fast ions approach the limit of trapped particles, in which the fast ion density becomes discontinuous on a flux surface since the ions can't reach the high field side in the magnetic mirror. In such cases, Eq (7) diverges and becomes invalid for these trapped particles. In this work, we will focus on the passing fast ions in reactive EGAMs and will not pursue an improved description for the trapped particles. The perpendicular direction of Eq. (6) is used to determine $V_{f, d}$, giving that

$$
\begin{array}{r}
\boldsymbol{V}_{f, \text { mag }}=-\frac{m V_{f \|}^{2}+p_{\perp f, \delta^{0}} / n_{f, \delta^{0}}}{e B_{0} R_{0}}\left(-R_{0} \hat{\boldsymbol{b}} \times \boldsymbol{\kappa}\right) \\
\approx \bar{V}_{f, \operatorname{mag}}\left(\hat{\boldsymbol{e}}_{\boldsymbol{r}} \sin \theta+\boldsymbol{e}_{\boldsymbol{\theta}} \cos \theta\right)+O\left(\epsilon^{2} \delta\right),
\end{array}
$$

where $\boldsymbol{\kappa}=\hat{\boldsymbol{b}} \cdot \nabla \hat{\boldsymbol{b}}$ is the field line curvature. We have used the vacuum field assumption $\nabla \times \boldsymbol{B}=0$. Taking a flux surface average, the numerator of $\bar{V}_{f, \text { mag }}$ is approximated by $\left(2-\Lambda_{0}\right) E_{0}$. Therefore, we reach $\bar{V}_{f, \operatorname{mag}} / V_{f \|} \sim O(\epsilon \delta)$.

It is more convenient to write $n_{f, \delta^{l}}=\bar{n}_{f, \delta^{l}}(r)(\cos \theta)^{l}+O\left(\epsilon \delta^{l}\right)$ and thus we have the continuity equation $\nabla \cdot\left(n_{f} \boldsymbol{V}_{f}\right)=0$ recast into the hierarchy given by

$$
\bar{n}_{f, \delta^{l+1}}=\frac{1}{l+1} \frac{q R_{0} \bar{V}_{f, \operatorname{mag}}}{V_{f \|}}\left(\frac{d}{d r}-\frac{l}{r}\right) \bar{n}_{f, \delta^{l}}+O\left(\epsilon \delta^{l+1}\right),
$$

showing that $\bar{n}_{f, \delta^{l+1}} / \bar{n}_{f, \delta^{l}} \sim O(\delta)$. Finally, adding the contribution for all the orders of $\delta$, we will have the equilibrium density profile with the FOW effects. It will be more verbose to find the $O\left(\epsilon \delta^{l}\right)$ terms which are later used in our global theory. The details of these terms are given in Appendix A. We note that the inclusion of the diamagnetic current will not contribute to the continuity equation and thereby will not change the fast ion density profile, since the divergence of curl is zero naturally given the form of $\boldsymbol{J}_{\mathrm{dia}}=-\nabla \times p_{\perp} \hat{\boldsymbol{b}} / B$.

The corresponding solution of the equilibrium profile is justified by comparing to the fast ion guiding centre drift orbits, as shown in Fig.1, indicating a very good match between the constant density surface and the orbits as expected. The difference in the sign of $V_{f \|}\left(V_{f \|}>0\right.$ for co-passing and $V_{f \|}<0$ for counter-passing) leads to a different sign in $\bar{n}_{f, \delta}$, the first Fourier harmonic of the fast ion density on a flux surface, and therefore a outward/inward shift of the co/counter-passing density contour. As we will show later in the paper, the different sign in $\bar{n}_{f, \delta}$ results in a different growth rate for different direction of injection. The profiles we have used are $\bar{n}_{f, 0}=n_{0} \exp \left(-r^{2} / a^{2}\right)$ as the specified density profile and $q=3$ being a constant of radius, with $R_{0}=1.7 \mathrm{~m}, \epsilon=0.3, B=2 \mathrm{~T}, E_{0}=75 \mathrm{keV}$ and $\Lambda_{0}=0.5$, i.e. typical DIII-D beam parameters [1]. The density hierarchy is truncated at $O\left(\delta^{3}\right)$ level. Note that in Fig. 1 we have ignored the change of density in $\theta$ direction due to $\mu$ being a constant of motion. We have also ignored the effect of particle loss at the boundary.

\section{Linear perturbation treatment}

The GAMs are electrostatic modes with toroidal mode number $n=0$. We retain only the poloidal mode number $m=0$ part of the perturbed electrostatic potential 

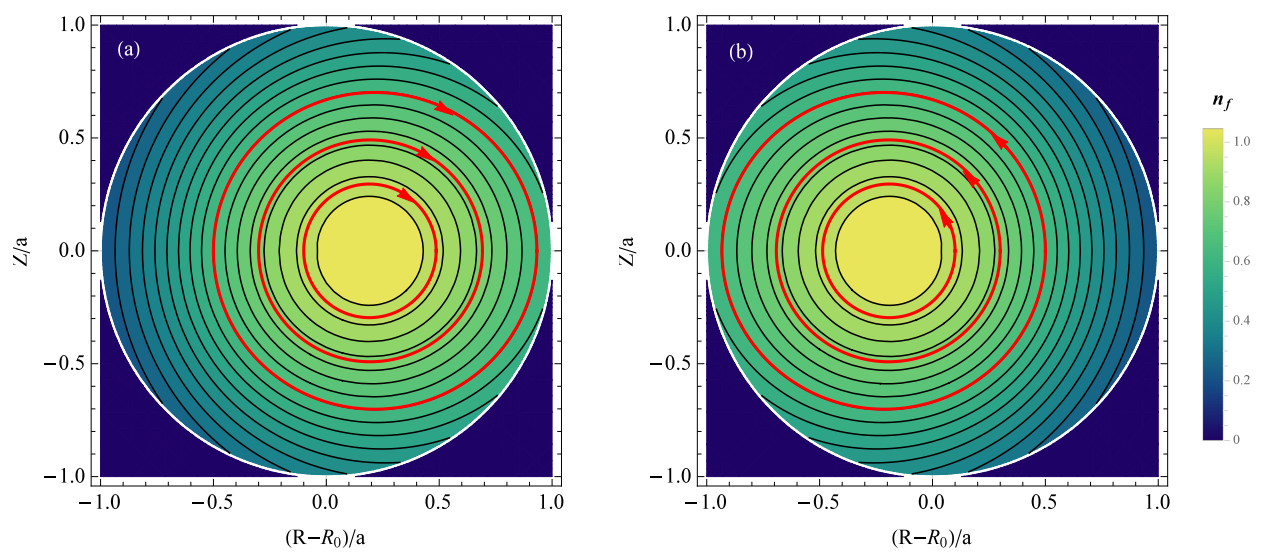

Figure 1. The equilibrium fast ion density $n_{f}$ contour on the plasma cross section, normalized to the on axis value, for co-passing (a) and counter-passing (b) flow direction. The fast ion guiding centre drift orbits for $E=75 \mathrm{keV}$ and $\Lambda=0.5$ are overplotted in red lines, with particles released at $r / a=0.1,0.3$ and 0.5 (inbound/outbound for co/counter-passing ions), from the most inner to the most outer orbit.

and two sidebands with $m= \pm 1$, written as

$$
\tilde{\Phi}=\tilde{\Phi}^{m=0}(r) e^{-i \omega t}+\tilde{\Phi}^{m=1}(r) e^{i \theta-i \omega t}+\tilde{\Phi}^{m=-1}(r) e^{-i \theta-i \omega t},
$$

in which $\omega=\omega_{r}+i \gamma$ is the complex frequency and the tilde labels the perturbed quantities. The radial derivative of $\tilde{\Phi}$ give rise to the $\boldsymbol{E} \times \boldsymbol{B}$ drift velocity as

$$
\tilde{\boldsymbol{V}}_{E} \approx \frac{\tilde{E}_{r} \hat{\boldsymbol{e}}_{\boldsymbol{r}} \times \boldsymbol{B}}{B^{2}} \approx \frac{\partial_{r} \tilde{\Phi}^{m=0}}{B} e^{-i \omega t} \hat{\boldsymbol{\pi}}=\tilde{V}_{E}(r) e^{-i \omega t} \frac{B_{0}}{B} \hat{\boldsymbol{\pi}}
$$

where we have used $\tilde{\Phi}^{m=0} / \tilde{\Phi}^{m= \pm 1} \gg 1$, an assumption that we will justify later. The $\boldsymbol{E} \times \boldsymbol{B}$ drift velocity is considered as the $O(1)$ velocity on which our argument of order is based. The dynamics of the system is determined by the linearized momentum equation of each species "s", given by

$$
\begin{aligned}
& m_{s} n_{s}\left(\frac{\partial \tilde{\boldsymbol{V}}_{s}}{\partial t}+\frac{\tilde{n}_{s}}{n_{s}} \boldsymbol{V}_{s} \cdot \nabla \boldsymbol{V}_{s}+\boldsymbol{V}_{s} \cdot \nabla \tilde{\boldsymbol{V}}_{s}+\tilde{\boldsymbol{V}}_{s} \cdot \nabla \boldsymbol{V}_{s}\right) \\
& =n_{s} e\left(-\nabla \tilde{\Phi}+\tilde{\boldsymbol{V}}_{s} \times \boldsymbol{B}\right)-\nabla \cdot\left[\tilde{p_{\perp s}} \overline{\boldsymbol{I}}+\left(\tilde{p}_{\|_{s}}-\tilde{p_{\perp s}}\right) \hat{\boldsymbol{b}} \hat{\boldsymbol{b}}\right] .
\end{aligned}
$$

We have used the electrostatic approximation, setting the perturbed magnetic field to be zero. We will also need the continuity equation of each species, given by

$$
\frac{\partial \tilde{n}_{s}}{\partial t}=-\nabla \cdot\left(n_{s} \tilde{\boldsymbol{V}}_{s}+\tilde{n}_{s} \boldsymbol{V}_{s}\right) .
$$

\subsection{Bulk plasma}

The perturbed fluid velocity of bulk ions consists of both the perpendicular and parallel components, written as

$$
\begin{gathered}
\tilde{\boldsymbol{V}}_{i}=\left[\tilde{V}_{i \|}^{m=1}(r) e^{i \theta-i \omega t}+\tilde{V}_{i \|}^{m=-1}(r) e^{-i \theta-i \omega t}\right] \hat{\boldsymbol{b}}+\tilde{\boldsymbol{V}}_{i, \delta} \\
+\tilde{V}_{E}(r) \frac{B_{0}}{B} e^{-i \omega t} \hat{\boldsymbol{\pi}},
\end{gathered}
$$


in which $\tilde{V}_{i \|}^{m= \pm 1}$ is the $O(1)$ parallel velocity and $\tilde{\boldsymbol{V}}_{i, \delta}$ the $O(\delta)$ velocity accounting for the magnetic, polarization and diamagnetic drift. The response of ion pressures, $\tilde{p_{\|}}$and $\tilde{\perp_{i}}$, are described by the double-adiabatic (CGL) fluid closure, given by

$$
\begin{gathered}
\frac{d \tilde{p}_{\|_{i}}}{d t}=-p_{i} \nabla \cdot \tilde{\boldsymbol{V}}_{i}-2 p_{i} \hat{\boldsymbol{b}} \cdot\left(\hat{\boldsymbol{b}} \cdot \nabla \tilde{\boldsymbol{V}}_{i}\right), \\
\frac{d \tilde{p_{\perp}}}{d t}=-2 p_{i} \nabla \cdot \tilde{\boldsymbol{V}}_{i}+p_{i} \hat{\boldsymbol{b}} \cdot\left(\hat{\boldsymbol{b}} \cdot \nabla \tilde{\boldsymbol{V}}_{i}\right) .
\end{gathered}
$$

For bulk ions, it is sufficient to keep only $\tilde{\boldsymbol{V}}_{E}$, the $\boldsymbol{E} \times \boldsymbol{B}$ drift velocity in the perturbed perpendicular velocity and ignore the higher order term $\tilde{\boldsymbol{V}}_{i, \delta}$, i.e. bulk ion FOW and FLR effects are ignored. Using Eq. (2) and extending $\tilde{p_{\|_{i}}}$ and $\tilde{p_{\perp_{i}}}$ similarly into poloidal Fourier harmonics, we obtain to the zeroth order of $\epsilon,{\tilde{\|_{\|}}}_{i}^{m=0}={\tilde{p_{\perp}}}_{s=0}^{m=0}=0$ and

$$
\begin{aligned}
& {\tilde{p_{\|}}}_{i}^{m= \pm 1}= \pm \frac{2 p_{i}}{R_{0} \omega} \tilde{V}_{E} \pm \frac{k}{\omega} 3 p_{i} \tilde{V}_{i \|}^{m= \pm 1}, \\
& {\tilde{p_{\perp}}}_{i}^{m= \pm 1}= \pm \frac{3 p_{i}}{2 R_{0} \omega} \tilde{V}_{E} \pm \frac{k}{\omega} p_{i} \tilde{V}_{i \|}^{m= \pm 1},
\end{aligned}
$$

in which $k=1 / q R_{0}$.

The ion density perturbation is given by the continuity equation Eq. (13). Again using Eq. (2) and retaining the zeroth order terms in $\epsilon$, the $m= \pm 1$ harmonics of the ion density perturbation are given by

$$
\tilde{n}_{i}^{m= \pm 1}= \pm \frac{n_{i}}{R_{0} \omega} \tilde{V}_{E} \pm \frac{k}{\omega} n_{i} \tilde{V}_{i \|}^{m= \pm 1},
$$

and $\tilde{n}_{i}^{m=0}=0$.

Since the electron transit frequency is much higher than the frequency of the mode, the response of electron is assumed to be isothermal (alias adiabatic in kinetic theory), which means $\tilde{p}_{e}=\tilde{n}_{e} T_{e}$, with $\tilde{p}_{e}$ and $\tilde{n}_{e}$ the perturbed electron pressure and density, respectively. Ignoring electron inertia, the momentum equation of electron gives

$$
0=n_{e} e \nabla \tilde{\Phi}-n_{e} e \tilde{\boldsymbol{V}}_{E} \times \boldsymbol{B}-T_{e} \nabla \tilde{n}_{e},
$$

where $\tilde{\boldsymbol{V}}_{e}$ is the perturbed electron velocity. The parallel direction of Eq. (20) gives

$$
\tilde{n}_{e}^{m= \pm 1}=e n_{e} \frac{\tilde{\Phi}^{m= \pm 1}}{T_{e}} .
$$

The quasi-neutrality condition is given by

$$
\tilde{n}_{e}^{m= \pm 1}=\tilde{n}_{i}^{m= \pm 1}+\tilde{n}_{f}^{m= \pm 1}
$$

with the perturbed electron pressure obtained by $\tilde{p}_{e}=\tilde{n}_{e} T_{e}$. Given the smallness of the fast ion density, the second term on the right hand side of Eq. (22) can often be ignored. Equating Eq. (19) and (21), one can obtain the following relationship: $\tilde{\Phi}^{m= \pm 1} / \tilde{\Phi}^{m=0} \sim O\left(\epsilon \delta \sqrt{T_{e} / E_{0}}\right)$ and $\tilde{\Phi}^{m= \pm 1}$ is thereby ignored in the $\boldsymbol{E} \times \boldsymbol{B}$ drift as stated earlier.

Finally, using Eq. (21) and (22) to eliminate $\tilde{E}_{\|}$, the parallel component of Eq. (12) in zeroth order of $\epsilon$ becomes

$$
m_{i} n_{i} \omega \tilde{V}_{i}^{m= \pm 1}= \pm k\left(\tilde{p}_{\|_{i}}^{m= \pm 1}+\tilde{n}_{i}^{m= \pm 1} T_{e}\right) .
$$


The perpendicular components of Eq. (12) can be rewritten into a sum of currents as

$$
\begin{aligned}
& \tilde{\boldsymbol{J}}_{i \perp, \delta}=\frac{1}{B}\left(\tilde{p_{\perp}}+\tilde{p}_{\|_{i}}+2 T_{e} \tilde{n}_{i}\right) \hat{\boldsymbol{b}} \times \boldsymbol{\kappa} \\
& -\nabla \times\left(\frac{\tilde{p_{\perp}}+T_{e} \tilde{n}_{i}}{B}\right)-i \omega \frac{m_{i} n_{i}}{B} \hat{\boldsymbol{b}} \times \tilde{\boldsymbol{V}}_{E},
\end{aligned}
$$

with the three terms on the right hand side accounting for the magnetic drift current, the diamagnetic current and the polarization current, respectively. A flux average quasi-neutrality condition,

$$
\langle\nabla \cdot \tilde{\boldsymbol{J}}\rangle=0
$$

can be imposed to obtain the dispersion relationship, in which $\tilde{\boldsymbol{J}}$ is the total current. The flux average simply cancels out the effect of parallel current and in the absence of the fast ions, Eq. (25) can simply be replaced by $\left\langle\nabla \cdot \tilde{\boldsymbol{J}}_{i \perp, \delta}\right\rangle=0$, with $\left\langle\nabla \cdot \tilde{\boldsymbol{J}}_{i \perp, \delta}\right\rangle$ given by

$$
\left\langle\nabla \cdot \tilde{\boldsymbol{J}}_{i \perp, \delta}\right\rangle=\frac{1}{r} \frac{\partial}{\partial r} r\left[i \omega \frac{m_{i} n_{i}}{B_{0}} \tilde{V}_{E}-\frac{i}{2 B_{0} R_{0}}\left(\tilde{p}_{\text {bulk }}^{m=1}-\tilde{p}_{\text {bulk }}^{m=-1}\right)\right],
$$

where $\tilde{p}_{b u l k}^{m= \pm 1}=\left(\tilde{\perp_{\perp}}+\tilde{p_{i}}+2 T_{e} \tilde{n}_{i}\right)^{m= \pm 1}$.

In the absence of fast ions, equating Eq. (26) to zero and using Eq. (17), (18), (19) and (23) yield a set of three equations with three unknowns $\tilde{V}_{E}, \tilde{V}_{i \|}^{m=1}$ and $\tilde{V}_{i \|}^{m=-1}$, defining an eigenvalue problem of $\omega$, i.e. the dispersion relationship. The dispersion relationship can be solved on each flux surface independently, giving three solutions corresponding to the thermal GAM, the ion sound wave and the low frequency zonal flow with the frequency from the highest to the lowest. The high- $q$ limit of the GAM frequency is given by

$$
\omega_{\mathrm{GAM}}^{2}(r)=\frac{2 T_{i}}{m_{i} R_{0}^{2}}\left(\frac{7}{4}+\tau_{e}\right)\left[1+O\left(q^{-2}\right)\right],
$$

in which $\tau_{e} \equiv T_{e} / T_{i}$ is the ratio of electron and ion temperature. Equation (27) recovers the gyrokinetic adiabatic index $\gamma_{e}=1$ and $\gamma_{i}=7 / 4$ by Sugama and Watanabe [26], with the couping to ion sound wave given by the $O\left(q^{-2}\right)$ term.

\subsection{Fast ions local theory}

We need to calculate the flux average perturbed radial current $\tilde{\boldsymbol{J}}_{f}$ for the fast ions and then use Eq. (25) to get the dispersion relationship. This calculation is carried on to the order of $O(\delta)$ for a local theory and $O\left(\delta^{3}\right)$ for a global theory, with the former discussed in this section.

We here define $\omega_{b}(r)=\left\langle V_{f \|}\right\rangle / q(r) R_{0}$ as the fast ion transit frequency. Similarly to the bulk ions, the $O(1)$ continuity equation can be simplified to

$$
\begin{gathered}
\tilde{n}_{f, \delta^{0}}^{m= \pm 1}= \pm \frac{1}{2 R_{0}\left(\omega \mp \omega_{b}\right)}\left[\bar{n}_{f, \delta^{0}}\left(1-c_{f}\right)+\bar{n}_{f, \delta} \frac{R_{0}}{r}\right] \tilde{V}_{E} \\
\pm \frac{k}{\omega \mp \omega_{b}} \bar{n}_{f, \delta^{0}} \tilde{V}_{f \|, \delta^{0}}^{m= \pm 1} .
\end{gathered}
$$

Note that we have retained the $O(\delta / \epsilon)$ term proportional to $n_{f, \delta}$, which can be an $O(1)$ contribution given the smallness of $\epsilon$. Given the form of the distribution function Eq. (3), the fluid closure is simplified to

$$
\tilde{p_{\perp}} \approx \Lambda_{0} E_{0} \tilde{n}_{f}, \quad \tilde{p}_{\|_{f}}=0 .
$$


The parallel direction of the momentum equation Eq. (12) yields the $O(1)$ equations for perturbed parallel velocity, given by

$$
\tilde{V}_{f \|, \delta^{0}}^{m= \pm 1}= \pm \frac{1+c_{f}}{2\left(\omega \mp \omega_{b}\right)} \omega_{b} q \tilde{V}_{E},
$$

in which we have ignored the contribution from the responding electrons balancing the parallel electric field, due to the argument that $T_{e} \ll E_{0}$ so the fast ion response will dominate over the electrons. The magnetic drift current, derived from the perpendicular direction of Eq. (12), has the form

$$
\tilde{\boldsymbol{J}}_{f \perp \mathrm{mag}, \delta}=\frac{1}{B}\left[{\tilde{p_{\perp}, \delta^{0}}}+m_{i} \tilde{n}_{f, \delta} V_{f \|}^{2}+2 m_{i} n_{f, \delta^{0}} V_{f \|} \tilde{V}_{f \|, \delta}\right] \hat{\boldsymbol{b}} \times \boldsymbol{\kappa},
$$

while the polarization current is simply given by

$$
\tilde{\boldsymbol{J}}_{f \perp p, \delta}=-i \omega \frac{m_{i} n_{f, \delta^{0}}}{B} \hat{\boldsymbol{b}} \times \tilde{\boldsymbol{V}}_{E} .
$$

We will ignore the diamagnetic current since it does not contribute to the divergence. Adding the current contributions from the bulk ions and fast ions and taking the quasi-neutrality condition Eq. (25), we reach the dispersion relationship, written as

$$
D(\omega, r)=1-[1-\alpha(r)] \frac{\omega_{\mathrm{GAM}}^{2}(r)}{\omega^{2}}-\alpha(r) G(\omega, r),
$$

in which $\alpha=\bar{n}_{f, \delta^{0}} /\left(n_{i}+\bar{n}_{f, \delta^{0}}\right)$ is the fast ion density proportion and $G(\omega, r)$ is the contribution from the fast ions. We have taken the high- $q$ limit and eliminate the contribution from the bulk ion parallel velocity. With the distribution function described by Eq. (3), the form of $G(\omega, r)$ is given by

$$
\begin{aligned}
G(\omega, r)=\frac{1+c_{f}}{2} & {\left[\left(3-c_{f}\right)+\frac{R_{0}}{r} \frac{\bar{n}_{f, \delta}}{\bar{n}_{f, \delta^{0}}}\right] } \\
& \times \frac{\omega_{b}^{2} q^{2}}{\omega^{2}-\omega_{b}^{2}}+\left(1+c_{f}\right)^{2} \frac{\omega_{b}^{4} q^{2}}{\left(\omega^{2}-\omega_{b}^{2}\right)^{2}},
\end{aligned}
$$

where $\omega_{b}$ and $q$ are functions of $r$. We note that $c_{f}=\Lambda_{0} /\left(2-2 \Lambda_{0}\right)$ from Eq. (7). In the limit $\Lambda_{0} \rightarrow 0$, i.e. completely tangential beam, $c_{f}=0$ and Eq. (34) reduces to the form derived from a bump-on-tail fast ion distribution with ignorable temperature in $\mathrm{Qu}$ et al [18] and was studied there in detail. While not taken into account in $\mathrm{Qu}$ et al , the additional term proportional to $\bar{n}_{f, \delta}$ originates from the density change on a flux surface due to FOW effects, and is responsible for the distinction between the co-passing and counter-passing injections. We will not study these effects separately here in a local theory, but will study it along with the global theory later in this work. Note that similar interpretation of this additional term can also be found in the work by Berk and Zhou [17].

\subsection{Fast ion global theory}

In the absence of bulk ion and fast ion FLR/FOW effects, the perturbations on different flux surfaces cannot communicate to each other and will oscillate at their own local GAM/EGAM frequency. Any global perturbation will disperse quickly into a radially highly oscillating structure and became highly damped due to phase mixing [27]. When fast ion FOW effects are taken into account, the drift orbits of the fast ions act as bridges between flux surfaces and allow the radial propagation of the mode. To take into account the fast ion FOW effects, we carry on the calculation of the fast 
ion radial current to $O\left(\delta^{3}\right)$. We note that the fast ion polarization drift current and diamagnetic current are ignored for higher order calculation. The contribution from the fast ion polarization drift current is a factor of $\alpha$ smaller than the bulk ion one, while the latter is comparable to the bulk plasma and thus the fast ion magnetic drift current, if the fast ion beta $\beta_{f} \sim \beta_{b u l k}$ the bulk plasma beta. The fast ion diamagnetic current, whose divergence vanishes and will not contribute to the continuity equation and $\nabla \cdot \boldsymbol{J}$, is also ignored.

The calculation is straight forward, by solving order by order the continuity equation and the momentum equation along with the closure condition Eq. (29). The corresponding equilibrium/perturbed variables and their ordering are listed in Table 1. We substitute Eq. (31) into the $O(\delta)$ continuity equation Eq. (13) to obtain $\tilde{n}_{f, \delta}$, while $\tilde{V}_{f \|, \delta}$ is calculated from the parallel direction of the momentum equation Eq. (12). The perpendicular direction of Eq. (12), is used in turn to get $\tilde{\boldsymbol{V}}_{f \perp \mathrm{mag}, \delta^{2}}$ of an order higher. This procedure is repeated to obtain $\tilde{\boldsymbol{V}}_{f \perp \mathrm{mag}, \delta^{3}}$ which will be used in Eq. (25). All the steps above have retained only the zeroth order terms in $\epsilon$. The detail of all the algebra involved is provided in Appendix B.

Table 1. The equilibrium/perturbed fast ion quantities and their order in $\delta$

\begin{tabular}{c|cccc}
\hline & $n_{f}$ & $\tilde{n}_{f}$ & $\boldsymbol{V}_{f}$ & $\tilde{\boldsymbol{V}}_{f}$ \\
\hline$O(1)$ & $n_{f, \delta^{0}}$ & $\tilde{n}_{f, \delta^{0}}$ & $V_{f \|} \hat{\boldsymbol{b}}$ & $\tilde{\boldsymbol{V}}_{E}+\tilde{V}_{f \|, \delta^{0}} \hat{\boldsymbol{b}}$ \\
$O(\delta)$ & $n_{f, \delta}$ & $\tilde{n}_{f, \delta}$ & - & $\tilde{V}_{f \|, \delta} \hat{\boldsymbol{b}}$ \\
$O\left(\delta^{2}\right)$ & $n_{f, \delta^{2}}$ & $\tilde{n}_{f, \delta^{2}}$ & - & $\tilde{V}_{f \|, \delta^{2}} \hat{\boldsymbol{b}}$ \\
$O\left(\delta^{3}\right)$ & $n_{f, \delta^{3}}$ & $\tilde{n}_{f, \delta^{3}}$ & - & - \\
$O(\epsilon \delta)$ & - & - & $\boldsymbol{V}_{f, \mathrm{mag}}$ & $\tilde{\boldsymbol{V}}_{f \perp \mathrm{mag}, \delta}$ \\
$O\left(\epsilon \delta^{2}\right)$ & - & - & - & $\tilde{\boldsymbol{V}}_{f \perp \mathrm{mag}, \delta^{2}}$ \\
$O\left(\epsilon \delta^{3}\right)$ & - & - & - & $\tilde{\boldsymbol{V}}_{f \perp \mathrm{mag}, \delta^{3}}$ \\
\hline
\end{tabular}

The dispersion relationship, after taken into account the $O\left(\delta^{3}\right)$ contributions, is now simplified to

$$
\begin{aligned}
\frac{d}{d r} \rho_{\|}^{2} \frac{1}{r}\left(F_{1} \frac{d}{d r} B_{1}\right. & \left.+F_{2} \frac{d}{d r} B_{2}\right) r \bar{n}_{f, \delta^{0}} \tilde{V}_{E} \\
& +\rho_{\|}^{2} \frac{1}{r^{2}}\left(F_{3} \frac{d}{d r} B_{3}+F_{4} \frac{d}{d r} B_{4}\right) r^{2} \frac{\bar{n}_{f, \delta^{0}}^{\prime} \tilde{V}_{E}}{\omega_{b}} \\
& +\rho_{\|}^{2} F_{5} \tilde{V}_{E}+n_{\text {total }} D(\omega, r) \tilde{V}_{E}=0,
\end{aligned}
$$

in which the brackets in the first and second terms are considered as differential operators and apply to the terms followed. The coefficients are given by

$$
\begin{aligned}
& F_{1}(\omega, r)=\frac{1}{8} \frac{\omega_{b}^{4} q^{2}}{\omega^{2}}\left(\frac{4}{\omega^{2}-4 \omega_{b}^{2}}-\frac{1}{\omega^{2}-\omega_{b}^{2}}\right), \\
& F_{2}(\omega, r)=\frac{1}{4} \frac{\omega_{b}^{6} q^{2}}{\omega^{2}}\left[\frac{16}{\left(\omega^{2}-4 \omega_{b}^{2}\right)^{2}}-\frac{1}{\left(\omega^{2}-\omega_{b}^{2}\right)^{2}}\right],
\end{aligned}
$$

and

$$
\begin{aligned}
& B_{1}(r)=\left(7-c_{f}+\frac{n_{f, \delta}}{n_{f, \delta^{0}}} \frac{R_{0}}{r}\right)\left(1+c_{f}\right)^{3}, \\
& B_{2}=\left(1+c_{f}\right)^{4},
\end{aligned}
$$


with all other terms given in Appendix C.

\section{Solving the dispersion relationship}

The global dispersion relationship Eq. (35) is solved numerically using a shooting method. The boundary condition we've used is $\tilde{V}_{E}(0)=0$ and the outgoing wave condition at the other end, given the experimentally observed outward propagating behaviour [28]. This outgoing boundary condition is achieved by introducing a perfectly matched layer [29] outside $r=a$ which strongly absorbs the outgoing wave and allows no reflection. In the perfectly matched layer, we preform the substitution

$$
\frac{d}{d r} \rightarrow \frac{1}{1+i \sigma_{0}(r / a-1)^{2}} \frac{d}{d r},
$$

and enforce zero Dirichlet boundary condition $\tilde{V}_{E}(b)=0$ at $b>a$. The value of $\sigma_{0}$ and $b$ vary from case to case. They are carefully chosen to be large enough to reach the convergence of the frequency, whilst finite to prevent ill-behaved solutions. Typical values are $\sigma_{0}=40$ and $b=1.2 a$. Based on this numerical scheme, we will perform a parameter scan on various profiles.

We will first explore the dependency of the global modes on the $q$ profile. Figure 2 (a) shows the two quadratic $q$ profiles we use, having monotonic and reversed shear respectively. In both cases $q_{\min }=3$, but the minimum is reached on axis for the monotonic case and at $r / a=0.4$ for the reversed shear case. The bulk plasma temperature profile used is $T_{e}=T_{i}=1-r^{2} / a^{2}$ in the unit of $\mathrm{keV}$, while the bulk density is taken to be constant. The zeroth order fast ion density profile is given by $\alpha(r)=0.1 \exp \left(-r^{2} / a^{2}\right)$ with the on axis ratio $10 \%$. These profiles are plotted in Fig.2 (b). Note that the relative high density of the fast ions is a requirement for our small orbit width expansion which will be explained later. For similar reason, we have chosen $B_{0}=3 \mathrm{~T}$, instead of $B_{0}=2 \mathrm{~T}$ for DIII-D, reducing the orbit width from $L_{\text {orbit }} / a=0.12$ to 0.08 . Other parameters we've used are $R_{0}=1.7 m, \epsilon=0.3, E_{0}=75 \mathrm{keV}, \Lambda_{0}=0.5$ and deuterium, typical DIII-D beam parameters. The direction of the fast ions is counter-passing.
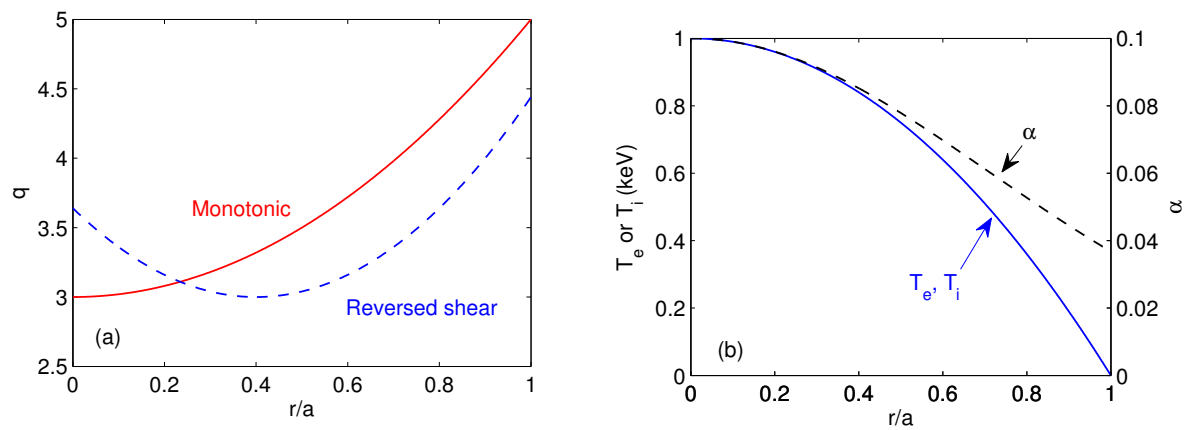

Figure 2. (a) The $q$ profile used in the monotonic (red solid line) and reversed shear (blue dashed line) case. (b) The $T_{e}$ and $T_{i}$ profile and the $\alpha$ profile. 


\subsection{Monotonic shear case}

The radial mode structure, $\tilde{V}_{E}$ as a function of minor radius, is plotted in Fig.3 (a) for the most unstable solution in the monotonic shear case. The frequency and growth rate are $32.7 \mathrm{kHz}$ and $57 \%$. The absolute amplitude is maximized at around $r / a=0.3$ and decays outward, showing a outgoing wave pattern (as a result of our boundary conditions). The direction of propagation can be understand by assuming $\tilde{V}_{E}(r) \sim \exp \left(i k_{r} r\right)$, and therefore the peak of $\Re\left(\tilde{V}_{E}\right) \sim \cos k_{r} r$ will be ahead of $\Im\left(\tilde{V}_{E}\right) \sim \cos k_{r} r$ by a quarter of wavelength if $k_{r}>0$, in agreement with Fig.2(a). By inspection of Fig.3 (a), $L_{\text {mode }}$ is a function of radial position. Instead of using the original definition in Eq. (1), we measure $L_{\text {mode }}$ by the full width at half maximum separately for the real and imaginary part and then takes an average. In the core region, $L_{\text {mode }}$ is measure between the first two nodes, giving $L_{\text {wave, core, while }} L_{\text {wave,edge }}$ is measured between the last two nodes. In addition, $L_{\text {orbit }}$ is measured at $r=0$ and 1 for the core and the edge, respectively. For Fig.3 (a), $L_{\text {mode, core }} / a=0.25$ with $\delta=0.32$. Our small parameter expansion is thus barely valid in the core. However, in the edge region $\delta \sim 1$, and our assumption is not valid. We would expect the inclusion of higher order corrections in $\delta$ to reduce the mode amplitude at the edge, since when the orbit width is comparable to the radial wavelength, the large drift orbit tends to "average out" the field, leading to a lower fast ion response.
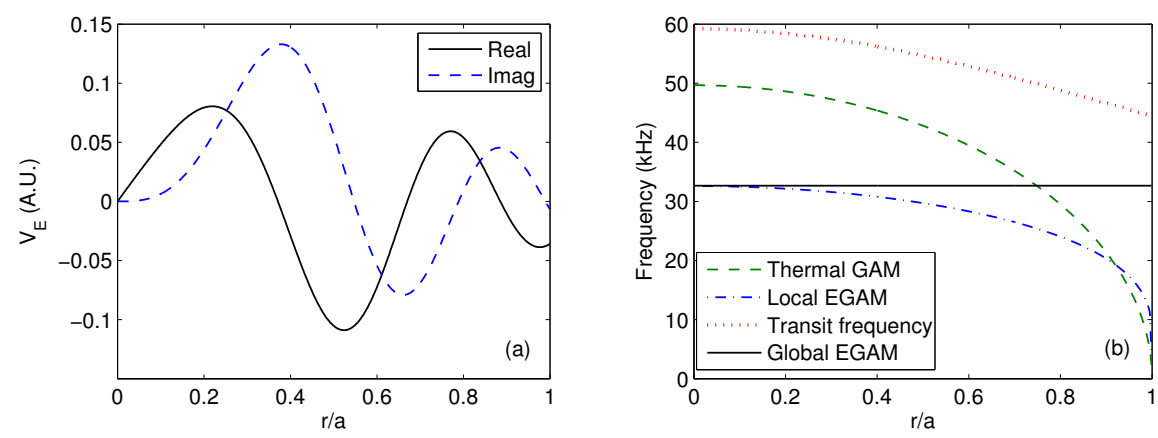

Figure 3. (a) The radial mode structure of the monotonic shear case. (b) The thermal GAM continuum, the transit frequency as a function of radius, the local EGAM frequency and the global EGAM frequency for the monotonic shear case.

The thermal GAM continuum, the frequency of the EGAM local solution, the transit frequency and the global EGAM frequency are plotted in Fig.3 (b). The thermal GAM continuum frequency peaks on axis and monotonically decreases to zero at the edge following the temperature profile. The frequency of the local EGAM is also monotonic with an on-axis extremum, mainly due to the monotonic $q$ profile and thus a monotonic fast ion transit frequency. The frequency of the global mode is $0.1 \mathrm{kHz}$ above the extremum, with an growth rate lower than the local solution (64\%) by $7 \%$.

To understand the property of the global solution, we expand the local dispersion relationship around its on-axis extremum. If we explore the case $L_{\text {mode }} \ll a$, the radial change of the equilibrium profiles becomes unimportant compared to the mode structure, and thus, our global dispersion relationship Eq. (35) can be simplified to

$$
\frac{d}{d r} \frac{1}{r} \frac{d}{d r} r \tilde{V}_{E}(r)+\left(p r^{2}+h \Delta \omega\right) \tilde{V}_{E}(r)=0,
$$


where $\Delta \omega=\omega-\omega_{\mathrm{EGAM}}(r=0)$, with $p$ and $h$ given by

$$
\begin{aligned}
& p=\frac{\partial^{2} D}{\partial r^{2}} /\left[\alpha \rho_{\|}^{2}\left(F_{1} B_{1}+F_{2} B_{2}\right)\right], \\
& h=\frac{\partial D}{\partial \omega} /\left[\alpha \rho_{\|}^{2}\left(F_{1} B_{1}+F_{2} B_{2}\right)\right],
\end{aligned}
$$

taken the value on axis and $\omega=\omega_{\mathrm{EGAM}}(r=0)$. The solution of Eq. (41), satisfying the zero Dirichlet boundary condition on axis, is given by

$$
\tilde{V}_{E}(r)=\frac{1}{r} e^{\frac{1}{2} i \sqrt{p} r^{2}} L_{\frac{1}{4} i h \Delta \omega / \sqrt{p}}^{-1}\left(i \sqrt{p} r^{2}\right),
$$

in which $L_{n}^{\alpha}(z)$ is the generalized Laguerre function, a solution to the Laguerre's equation

$$
z y^{\prime \prime}(z)+(\alpha+1-z) y^{\prime}(z)+n y(z)=0 .
$$

The asymptotic behaviour of solution Eq. (44) at $+\infty$ is written as

$$
\begin{aligned}
\tilde{V}_{E}(r \rightarrow+\infty) \rightarrow & \frac{C_{1}(p, h \Delta \omega)}{\Gamma\left(-\frac{i h \Delta \omega}{4 \sqrt{p}}\right)} r^{-\frac{i h \Delta \omega}{2 \sqrt{p}}-1} e^{-\frac{1}{2} i \sqrt{p} r^{2}} \\
& +\frac{C_{2}(p, h \Delta \omega)}{\Gamma\left(\frac{i h \Delta \omega}{4 \sqrt{p}}\right)} r^{\frac{i p \Delta \omega}{\sqrt{h}}-1} e^{\frac{1}{2} i \sqrt{p} r^{2}},
\end{aligned}
$$

in which $\Gamma(z)$ is the Gamma function, and $C_{1}$ and $C_{2}$ are non-zero unless $p=0$. If $\Re(\sqrt{p})>0$, the first term in Eq. (46) represents an inward propagating component and the second term is an outward propagating one. To satisfy our outgoing boundary condition, we need the Gamma function in the denominator of the first term to approach infinity, i.e. $1 / \Gamma(z) \rightarrow 0$. Singularities happens for $\Gamma(z)$ when $z$ is a negative integer, leading to the eigenvalue condition

$$
\Delta \omega=\left\{\begin{array}{ll}
-4 i N \sqrt{p} / h & \text { if } \Re(\sqrt{p})>0, \\
4 i N \sqrt{p} / h & \text { if } \Re(\sqrt{p})<0,
\end{array} \quad N=1,2,3, \cdots .\right.
$$

For the case demonstrated in Fig. $3, \sqrt{p}=34.3+7.99 i, h=(7.3+1.1) \times 10^{-3}$, and the calculated frequency and growth rate from Eq. (47) with $N=1$ are given by $33.7 \mathrm{kHz}$ and $53.9 \%$. The deviation of the analytic solution from the numerical solution is a consequence of $L_{\text {mode }} \ll a$ being unsatisfied. We note that when $B_{0}$ is increased to $9 \mathrm{~T}$ and $L_{\text {mode }}$ is reduced to $0.15 a$, we have got a much better match (frequency $33.4 \mathrm{kHz}$ and growth rate $54.1 \%$ ).

Inspection of Eq. (44) shows $L_{\text {mode }}$ around the axis is approximately given by

$$
L_{\text {mode,core }} \sim p^{-1 / 4} \sim \alpha^{1 / 4} q^{1 / 2} L_{\text {orbit }}^{1 / 2} L_{\text {EGAM }}^{1 / 2} .
$$

Away from the core region, a WKB approximation can be applied since the length scale of the equilibrium quantities is comparable to the minor radius, while the mode width is comparable to $L_{\text {orbit }}$ and $L_{\text {orbit }} \ll a$. Given $\omega_{\text {GAM }}$ vanishes at the edge, $D(\omega, r) \sim 1$, and therefore

$$
L_{\text {mode,edge }} \sim \alpha^{1 / 2} q L_{\text {orbit }},
$$

matching our observations in Fig.3. Equation (49) also indicates that $\delta \ll 1$ is not satisfied at the plasma edge unless an unrealistic $\alpha$ is assumed.

A numerical parameter scan is performed to study the relationship between the orbit width and the mode width. The drift orbit width is changed by adjusting the 
field strength $B_{0}$, with the advantage of conserving the EGAM local frequency. As the field strength is increased, the radial model structure gradually changes from Fig.3 (a) to Fig.4 (a) with a shorter wavelength. Similar to the previous case, we measure the radial mode width by the full width of the real and imaginary parts at half maximum and takes an average, for $B_{0}$ from $3 \mathrm{~T}$ to $9 \mathrm{~T}$. The "measurement" is taken between

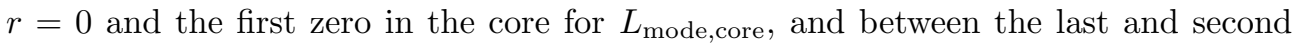
last zeros at the edge for $L_{\text {mode,edge }}$. The corresponding relationship between the orbit width and the mode width is shown in Fig.4 (b). The trend line of $L_{\text {mode }}$ in Fig.4 (b) matches Eq. (48) and (49) respectively in the core and at the edge.
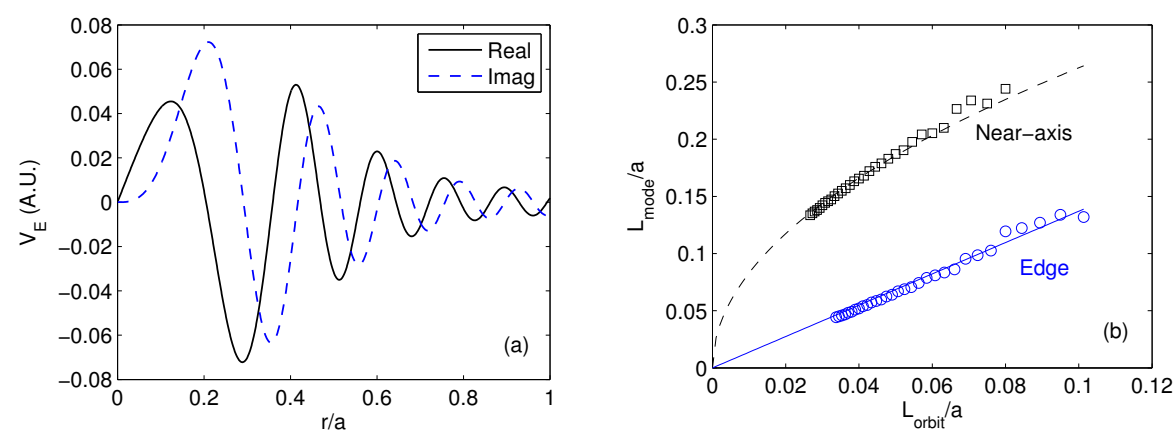

Figure 4. (a) The radial mode structure of the monotonic shear case with increased field strength $B_{0}=9 \mathrm{~T}$. (b) Scanning $B_{0}$ from $3 \mathrm{~T}$ to $9 \mathrm{~T}$, the radial mode width as a function of drift orbit width near the axis (black squares) and at the edge (blue circles). for the monotonic shear case, with a square root and a linear trend line, respectively.

\subsection{Reversed shear case}

We have repeated our mode structure calculation for the reversed shear case, with $\tilde{V}_{E}(r)$ and global/local EGAM frequency given in Fig.5. Inspection of Fig.5 (b) shows that the thermal GAM continuum is almost identical to the monotonic shear case, since the same temperature profile is used and the $O\left(q^{-2}\right)$ correction to the GAM frequency is eligible for $q \geq 3$. However, the local EGAM continuum in the two cases are qualitatively different. In the reversed shear case, an extremum is presented at $r / a=0.37$ near the $q=q_{\min }$ surface. This off-axis extremum is formed due to the extremum of $\omega_{b}=v_{\|} / q R_{0}$ the fast ion transit frequency at the same radius, and ultimately due to the shear reversal. The frequency of the most unstable global mode is $1 \mathrm{kHz}$ above this maximum at $33.3 \mathrm{kHz}$, with a growth rate of $61.5 \%$. Similar to the monotonic shear case, the mode structure is radially propagating with $\delta=0.29$ in core, but the mode amplitude is higher around the core region compared to the edge.

Again we can simplify Eq. (35) around the maximum of the EGAM local continuum, giving that

$$
\frac{d^{2}}{d r^{2}} \tilde{V}_{E}(r)+\left[p\left(r-r_{\mathrm{ex}}\right)^{2}+h \Delta \omega\right] \tilde{V}_{E}(r)=0,
$$

with the definition of $p$ and $h$ the same as Eq. (42) and (43), but evaluated at $r=r_{\mathrm{ex}}$ and $\omega=\omega_{\mathrm{ex}}$, labeling the radius and the complex frequency at the extremum, 

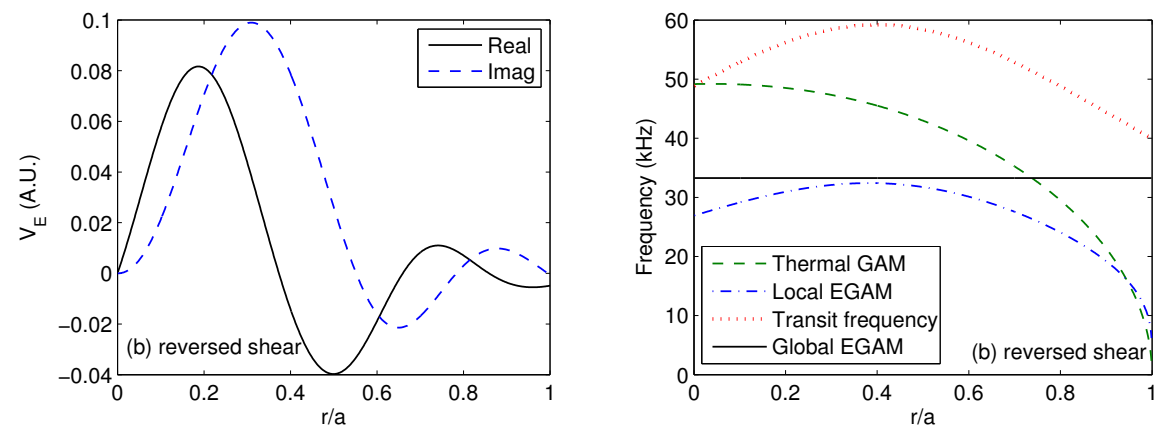

Figure 5. (a) The radial mode structure of the reversed shear case. (b) The thermal GAM continuum, the transit frequency as a function of radius, the local EGAM frequency and the global EGAM frequency for the reversed shear case.

respectively. The solution to Eq. (50) is given by

$$
\tilde{V}_{E}(r)=\left\{\begin{array}{ll}
D_{\nu}\left[\sqrt{2}(-p)^{1 / 4}\left(r-r_{\mathrm{ex}}\right)\right], & \Re(\sqrt{p})<0, \\
D_{-1-\nu}\left[-\sqrt{2} i(-p)^{1 / 4}\left(r-r_{\mathrm{ex}}\right)\right], & \Re(\sqrt{p})>0,
\end{array},\right.
$$

in which the parabolic cylinder function $D_{\nu}(z)$ is the solution to the Weber's equation

$$
y^{\prime \prime}(z)+\left(\nu+\frac{1}{2}-\frac{1}{4} z^{2}\right) y(z)=0,
$$

and

$$
\nu=-\frac{1}{2}-\frac{i h \Delta \omega}{2 \sqrt{p}} .
$$

Asymptotically, the solution in Eq. (51) satisfies

$$
\tilde{V}_{E}(r \rightarrow+\infty) \rightarrow\left\{\begin{array}{ll}
r^{\nu} e^{-\frac{1}{2} i \sqrt{p} r^{2}}, & \Re(\sqrt{p})<0, \\
r^{-\nu-1} e^{\frac{1}{2} i \sqrt{p} r^{2}}, & \Re(\sqrt{p})>0,
\end{array},\right.
$$

with the outgoing boundary condition satisfied. The parameter $\nu$, and thereby the mode frequency, will be determine by the other boundary condition $\tilde{V}_{E}(0)=0$.

The approximate solution Eq. (51) is no longer valid around the magnetic axis, as the $1 / r$ factor in the second order derivative term in Eq. (35) becomes important. Near the axis, the solution is given by

$$
\tilde{V}_{E}(r) \approx J_{1}(\sqrt{\beta} r), \quad \beta=\frac{D\left(\omega_{\mathrm{ex}}, r=0\right)}{\alpha \rho_{\|}^{2}\left(F_{1} B_{1}+F_{2} B_{2}\right)},
$$

where $J_{n}(z)$ is the Bessel function of the first kind and $\beta$ is evaluated on axis and at $\omega=\omega_{\text {ex }}$ so it does not depend on the choice of $\Delta \omega$. Theoretically, we can connect the core solution Eq. (55) and the outer region solution Eq. (51) at $r=r_{c}$. The connection criterion

$$
\left.\tilde{V}_{E}\right|_{r=r_{c}^{-}} ^{r=r_{c}^{+}}=\left.\frac{d \tilde{V}_{E}}{d r}\right|_{r=r_{c}^{-}} ^{r=r_{c}^{+}}=0,
$$

should define the eigenvalue problem of $\nu$ and therefore $\Delta \omega$. However, in practice, we've found that although the eigenfunction is insensitive to the choice of $r_{c}, \Delta \omega$ 
depends quite heavily on $r_{c}$. Therefore, we will not pursue a quantitative match between the full numerical solution and the analytic one in the reversed shear case.

One would expect that the scaling law Eq. (48) still holds for the reversed shear case in the core region given the form of the solution in Eq. (51). However, as we will show later, this scaling law fails for $B_{0}>4 \mathrm{~T}$. In Fig.6 (a), we have plotted the radial mode structure for a increased field strength $B_{0}=5 \mathrm{~T}$. The frequency of the mode is now $32.1 \mathrm{kHz}, 0.6 \mathrm{kHz}$ below the maximum of the continuuum. The mode structure becomes quite core-localized, with a low-amplitude propagating tail near the edge. An analogy can be made between Eq. (50) and the 1D Schrödinger equation in the quantum mechanics with

$$
E-V(r)=h \Delta \omega+p\left(r-r_{\mathrm{ex}}\right)^{2},
$$

in which $E$ is the energy and $V(r)$ is the potential. With $\Re[h]>0$ in our case, a global mode frequency lower than the EGAM extremum frequency means $E<V(r)$ near the extremum, a classically forbidden region. Near the magnetic axis and at the edge, we have $E>V(r)$ the classically allowed region. A mode excited in the core is allowed to propagate across the forbidden region through quantum tunneling and into the outer region. Therefore, we would expect a significantly reduced mode amplitude at the edge compared to the core since the lower the energy $E$ is, the less wave will "leak" into the outer region. Due to the observation that as $B_{0}$ increases, the global frequency becomes lower than the global continuum, the solution in Eq. (51), assuming an extremum mode, is no longer appropriate. As a result, the mode width will not follow Eq. (48) for large $B_{0}$, in the region where $\omega<\omega_{\text {ex }}$. This is confirmed in Fig.6 (b), in which we have plotted a scan of the mode width over the orbit width by increasing the field strength. For orbit width less than $0.06 a$, the curve is found to follow the trend line $\propto L_{\text {orbit }}$ instead of a square root, which can be derived from the on axis solution Eq. (55). For $L_{\text {orbit }}>0.06 a$, the frequency of the global mode is above the EGAM continuum and therefore the scaling law is changed back to the square root dependency predicted by Eq. (51). The mode width at the edge, on the other hand, gives a good match to Eq. (49), as shown by Fig.6 (b).
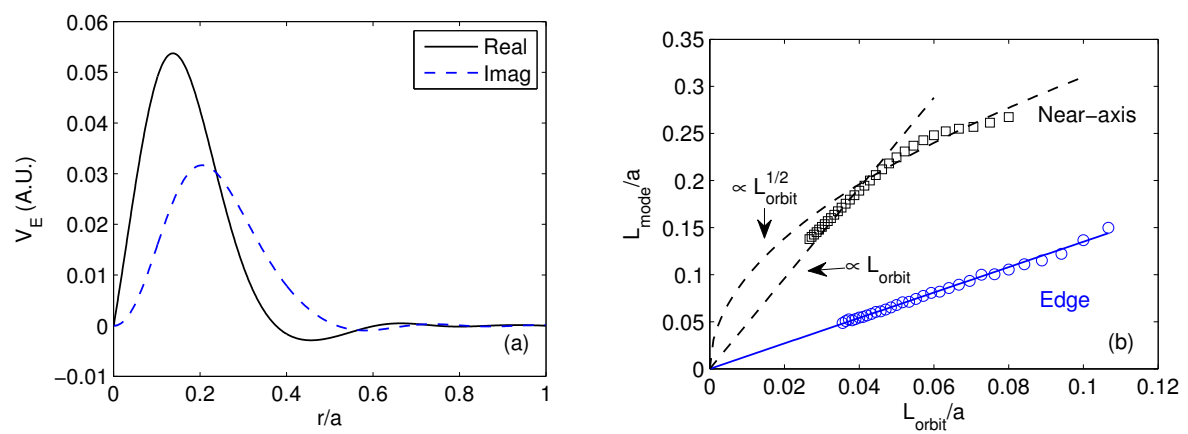

Figure 6. (a) The radial mode structure of the reversed shear case with increased field strength $B_{0}=5 \mathrm{~T}$. (b) Scanning $B_{0}$ from $3 \mathrm{~T}$ to $9 \mathrm{~T}$, the radial mode width as a function of drift orbit width near the axis (black squares) and at the edge (blue circles) for the reversed shear case with trend lines. 


\subsection{Dependency on injection direction}

In this section, the dependency of the mode frequency and growth rate on the injection direction is studied by changing the sign of $\omega_{b}$ (positive for co-passing and negative for counter-passing) and keeping all other parameters unchanged. Figure 7 shows a scan of the most unstable global mode frequency and growth rate as a function of the fast ion proportion for the monotonic shear scenario and the reversed shear scenario, but assuming either co-passing or counter-passing fast ions. The frequency of the mode in all the cases decreases as the fast ion density increases, while the mode is becoming more unstable at the same time, similar to the behaviour of the local solutions in $\mathrm{Qu}$ et al [18]. In fact, the complex frequency of the global mode is mainly determined by the local EGAM continuum as understood from the analysis in Section 4.1 and 4.2: for an extremum mode, $\omega=\omega_{\text {ex }}+\Delta \omega$ and $\Delta \omega$ is small. Looking back into the EGAM local dispersion relationship Eq. (33), the only distinction between the co/counter-passing ions comes from the additional term proportional to $\bar{n}_{f, \delta}$ in Eq. (34). As stated in Section 2, the sign of $\bar{n}_{f, \delta}$ will be different for co/counter-passing ions. All other terms come with $\omega_{b}^{2}$ and therefore the effect of direction is canceled, while $c_{f}$ is also identical for different injection directions. Therefore, we would expect this additional term, originated from the fact that counter-passing ions have inwardly shifted orbits and co-passing ions outward, to modify the EGAM local continuum, and reflects into the distinction between counter-passing ions and co-passing ions.
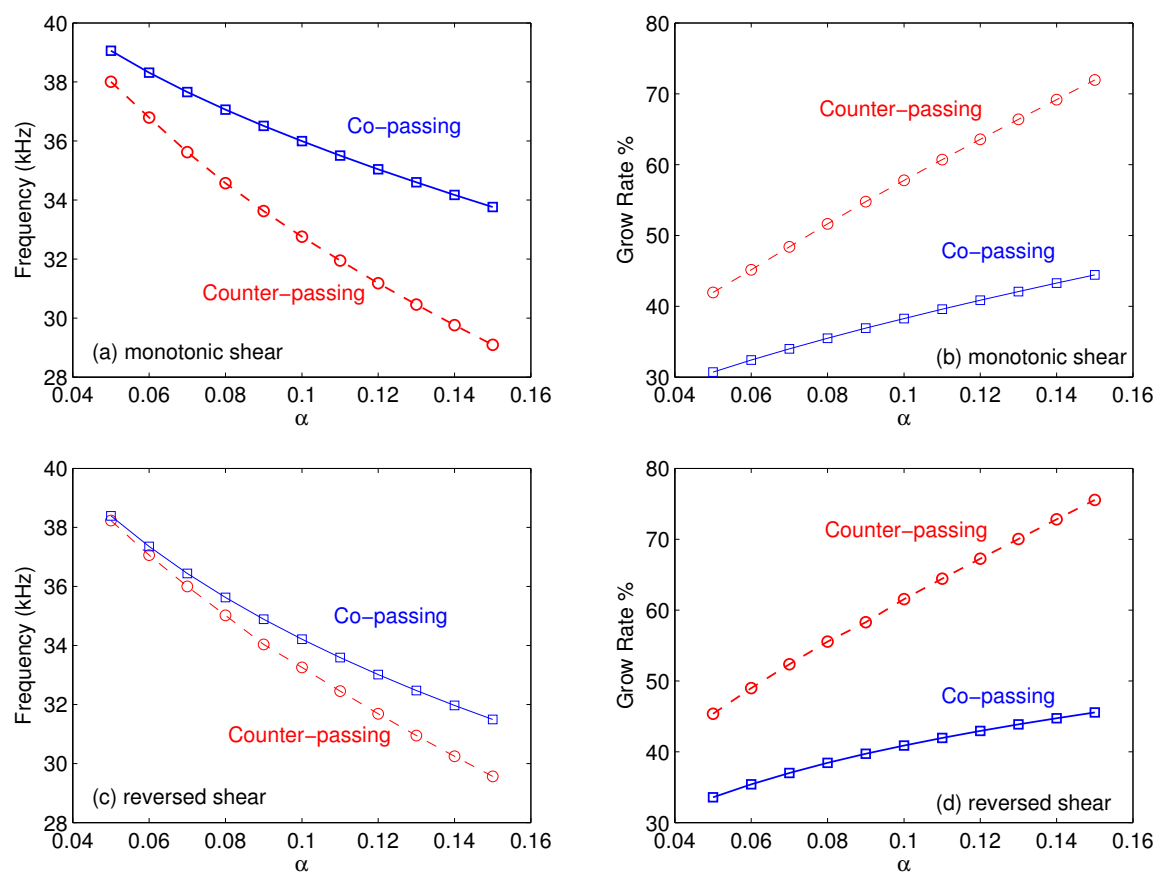

Figure 7. The frequency (a) and the growth rate (b) for the monotonic case, with counter-passing (red circles) and co-passing (blue squares) fast ions, scanned over fast ion proportion $\alpha$. (c)(d) The same scan for the reversed shear case. We have restricted our scan domain to $\alpha>0.05$ to satisfy the condition $L_{\text {mode }} \gg L_{\text {orbit }}$.

Figure 7 shows that the real frequency of the counter-passing mode is slightly 
below the co-passing mode in both the monotonic shear and reversed shear case. But more interestingly, the growth rate of the counter-passing mode is $50 \%$ larger than the co-passing one for $\alpha=0.05$. This difference enlarges to $100 \%$ when the fast ion density is $15 \%$ the total ion density, indicating that the reactive EGAMs in the presence of counter-passing ions is much more unstable than the one with co-passing ions given all other conditions the same. It is possible that in the presence of a strong damping (such as the ion Landau damping), the reactive EGAMs induced by co-passing ions are suppressed due to their relative lower growth rate, while in the counter-injection scenario, the growth rate is strong enough to overcome the damping. Our result is consistent with experimental observation that EGAMs are more often observed in plasmas with counter-injection $[1,28]$.

\section{Conclusion}

Extending the previous model for local reactive EGAMs, we have added the finite drift orbit width (FOW) effects to our three-fluid model under the assumption that $L_{\text {orbit }} \ll L_{\text {mode }}$, leading to a second order ODE as the dispersion relationship. The model is valid when the fast ion distribution function is beam-like: we have therefore used a single-energy single-pitch distribution, consistent with the scenario of early beam heating when the beam is not yet slowed down.

It has been found that with proper boundary conditions, a radially propagating structure can be formed when the $q$ profile is either monotonic or reversed shear. In the monotonic shear case, the global EGAM frequency is slightly higher than the local frequency at the extremum on axis, while the growth rate is lower than the local growth rate at the extremum. A qualitative analytic solution of the mode structure reveals the relationship $L_{\text {mode }} \propto L_{\text {orbit }}^{1 / 2}$ near the axis and $L_{\text {mode }} \propto L_{\text {orbit }}$ at the edge. This finding is later confirmed by a numerical scan which modifies $L_{\text {orbit }}$ by increasing the field strength, while keeping the EGAM local continuum unchanged. In the reversed shear case, for $L_{\text {orbit }}>0.06 a$, the global EGAM is also an extremum mode residing on the top of the local EGAM extremum at $r \approx 0.4 a$, the $q=q_{\min }$ surface. One would expect the relationship between $L_{\text {mode }}$ and $L_{\text {orbit }}$ for the monotonic case to be applicable in the reversed shear case. However, when $L_{\text {orbit }}<0.06 a$, the global EGAM frequency becomes lower than the value at the extremum. This resembles the case when the energy is lower than the top of the potential barrier in a quantum system, meaning that the mode must be localized at the core where it is excited, although "tunneling" to the other side of the barrier (the edge region) is permitted. In this case, $L_{\text {mode }}$ is found to scale linearly with $L_{\text {orbit }}$.

Based on the two cases with different $q$ profiles, we have also investigated the dependency of the global mode frequency and growth rate on the injection direction. It is shown that the global EGAM frequency in the presence of a counter-beam is slightly lower than the co-beam, but is significantly more unstable compared to the co-beam case. Given the same amount of damping, it is possible that the counterbeam reactive EGAM can encounter the damping while the co-beam one is suppressed, consistent with the observation that EGAMs are more likely to appear in counter-beam experiments.

In our future work, we plan to release the assumption $L_{\text {orbit }} \ll L_{\text {mode }}$ and takes into account the full drift orbit effects of the fast ions. This may involve the use of a non-local fluid closure. The comparison to existing kinetic codes with full drift orbit effects such as LIGKA [30] is also possible. In the perspective of validation, a survey of 
the mode frequency is needed for different injection directions, while the measurement of the mode structure can be available through spectroscopy with good temporal ( a few ms for beam turn on) and spatial resolution (sufficient for radial structure) such as soft x-ray [31]

\section{Acknowledgments}

The authors would like to thank Dr. A. Biancalani, Dr. G.Y. Fu, Prof. B.N. Breizman and Dr. B. Layden for fruitful discussions; Dr. M.A. Van Zeeland and Dr. R. Nazikian for DIII-D data. This work is funded by China Scholarship Council, the AINSE Postgraduate Research Award, Australian ARC project DP1093797 and FT0991899. This work was part funded by the RCUK Energy Programme [grant number EP/I501045].

\section{Appendix A. Higher order $O\left(\epsilon \delta^{l}\right)$ terms in the equilibrium density profile}

If we define

$$
n_{f, \delta^{l}} \approx \bar{n}_{f, \delta^{l}}\left[1-\left(1+c_{f, \delta^{l}}\right) \frac{r}{R_{0}} \cos \theta\right] \cos ^{l} \theta,
$$

after calculating the $O(\epsilon \delta)$ and $O\left(\epsilon \delta^{2}\right)$ component of the equilibrium continuity equation, we get that

$$
\begin{aligned}
& c_{f, \delta}=c_{f}+\frac{1}{2} \frac{\Lambda_{0}}{2-\Lambda_{0}} c_{f}, \\
& c_{f, \delta^{2}}=\frac{2}{2-\Lambda_{0}} c_{f},
\end{aligned}
$$

which will be used in further calculation in Appendix B.

\section{Appendix B. Detail derivation of the higher order fast ion drift current}

The parallel direction of Eq. (12) gives the equations for the parallel velocity. Retaining only the zeroth order terms in $\epsilon$, we have in $O(\delta)$ that

$$
\begin{aligned}
& \tilde{V}_{f \|, \delta}^{m= \pm 2}=\mp \frac{1}{2} \frac{\bar{V}_{f, \operatorname{mag}}}{\omega \mp 2 \omega_{b}}\left(\frac{d}{d r}-\frac{1}{r}\right) \tilde{V}_{f \|, \delta^{0}}^{m= \pm 1}, \\
& \tilde{V}_{f \|, \delta}^{m=0}=\frac{1}{2} \frac{\bar{V}_{f, \mathrm{mag}}}{\omega}\left(\frac{d}{d r}+\frac{1}{r}\right)\left(\tilde{V}_{f \|, \delta^{0}}^{m=1}-\tilde{V}_{f \|, \delta^{0}}^{m=-1}\right),
\end{aligned}
$$

and in $O\left(\delta^{2}\right)$ that

$$
\tilde{V}_{f \|, \delta^{2}}^{m= \pm 1}= \pm \frac{1}{2} \frac{\bar{V}_{f, \mathrm{mag}}}{\omega \mp \omega_{b}}\left[\left(\frac{d}{d r}+\frac{2}{r}\right) \tilde{V}_{f \|, \delta}^{m= \pm 2}-\frac{d \tilde{V}_{f \|, \delta}^{m=0}}{d r}\right] .
$$

The perpendicular direction of Eq. (12), if the polarization drift and the diamagnetic drift are excluded, gives in $O(\delta)$ that

$$
\begin{aligned}
\left(\nabla \cdot n \widetilde{\boldsymbol{V}_{f \perp \mathrm{mag}, \delta}}\right)^{m= \pm 2}= & \\
& \mp \frac{i}{2}\left(\frac{d}{d r}-\frac{1}{r}\right) \bar{V}_{f, \operatorname{mag}}\left(\tilde{n}_{f, \delta^{0}}^{m= \pm 1}+\bar{n}_{f, \delta^{0}} \frac{2}{1+c_{f}} \frac{\tilde{V}_{f \|, \delta^{0}}^{m= \pm 1}}{\omega_{b} q R_{0}}\right),
\end{aligned}
$$




$$
\begin{aligned}
\left(\nabla \cdot n \widetilde{\boldsymbol{V}_{f \perp \text { mag }, \delta}}\right)^{m=0} & =\frac{i}{2}\left(\frac{d}{d r}+\frac{1}{r}\right) \bar{V}_{f, \text { mag }} \\
& \left.\times\left(\tilde{n}_{f, \delta^{0}}^{m=1}+\bar{n}_{f, \delta^{0}} \frac{2}{1+c_{f}} \frac{\tilde{V}_{f \|, \delta^{0}}^{m= \pm 1}}{\omega_{b} q R_{0}}-\tilde{n}_{f, \delta^{0}}^{m=-1}-\bar{n}_{f, \delta^{0}} \frac{2}{1+c_{f}} \frac{\tilde{V}_{f \|, \delta^{0}}^{m= \pm 1}}{\omega_{b} q R_{0}}\right) \mathrm{B}, 5\right)
\end{aligned}
$$

in $O\left(\delta^{2}\right)$ that

$$
\begin{aligned}
\left(\nabla \cdot n \boldsymbol{V}_{f \perp \mathrm{mag}, \delta^{2}}\right. & )^{m= \pm 1}= \pm \frac{i}{2}\left(\frac{d}{d r}+\frac{2}{r}\right) \\
& \bar{V}_{f, \operatorname{mag}}\left(\tilde{n}_{f, \delta}^{m= \pm 2}+\bar{n}_{f, \delta^{0}} \frac{2}{1+c_{f}} \frac{\tilde{V}_{f \|, \delta}^{m= \pm 2}}{\omega_{b} q R_{0}}\right) \\
\mp & \frac{i}{2} \frac{d}{d r} \bar{V}_{f, \operatorname{mag}}\left(\tilde{n}_{f, \delta}^{m=0}+\bar{n}_{f, \delta^{0}} \frac{2}{1+c_{f}} \frac{\tilde{V}_{f \|, \delta}^{m=0}}{\omega_{b} q R_{0}}\right) \\
\mp & \frac{i}{1+c_{f}} \bar{V}_{f, \operatorname{mag}}\left(\frac{1}{2} \frac{d}{d r} \bar{n}_{f, \delta} \frac{\tilde{V}_{f \|, \delta^{0}}^{m=\mp 1}}{\omega_{b} q R_{0}}-\frac{1}{r} \bar{n}_{f, \delta} \frac{\tilde{V}_{f \|, \delta^{0}}^{m= \pm 1}}{\omega_{b} q R_{0}}\right),
\end{aligned}
$$

and finally in $O\left(\delta^{3}\right)$ that

$$
\begin{aligned}
\left(\nabla \cdot n \boldsymbol{V}_{f \perp \mathrm{mag}, \delta^{3}}\right)^{m=0} & =\frac{i}{2}\left(\frac{d}{d r}+\frac{1}{r}\right) \bar{V}_{f, \operatorname{mag}}\left[\tilde{n}_{f, \delta^{2}}^{m=1}-\tilde{n}_{f, \delta^{2}}^{m=-1}\right. \\
& +\frac{2}{1+c_{f}} \frac{1}{\omega_{b} q R_{0}}\left(\bar{n}_{f, \delta^{0}} \tilde{V}_{f \|, \delta^{2}}^{m=1}-\bar{n}_{f, \delta^{0}} \tilde{V}_{f \|, \delta^{2}}^{m=-1}\right. \\
& \left.\left.+\frac{1}{2} \bar{n}_{f, \delta} \tilde{V}_{f \|, \delta}^{m=2}-\frac{1}{2} \bar{n}_{f, \delta} \tilde{V}_{f \|, \delta}^{m=-2} \frac{1}{4} \bar{n}_{f, \delta^{2}} \tilde{V}_{f \|, \delta^{0}}^{m=1}-\frac{1}{4} \bar{n}_{f, \delta^{2}} \tilde{V}_{f \|, \delta^{0}}^{m=-1}\right)\right],
\end{aligned}
$$

which are used in the calculation of the perturbed density and

$$
\left\langle\nabla \cdot \tilde{\boldsymbol{J}}_{i \perp, \delta^{3}}\right\rangle=e\left(\nabla \cdot n \widetilde{\boldsymbol{V}_{f \perp \mathrm{mag}, \delta^{3}}}\right)^{m=0} .
$$

The perturbed density is calculated through the continuity equation Eq. (13), which converts to

$i\left(\omega \mp 2 \omega_{b}\right) \tilde{n}_{f, \delta}^{m= \pm 2}=$

$$
\begin{aligned}
& \left(\nabla \cdot n \widetilde{\boldsymbol{V}_{f \perp \mathrm{mag}, \delta}}\right)^{m= \pm 2} \pm i k\left(2 \bar{n}_{f, \delta} \tilde{V}_{f \|, \delta}^{m= \pm 2}+i k \bar{n}_{f, \delta} \tilde{V}_{f \|, \delta^{0}}^{m= \pm 1}\right) \\
& \pm \frac{i}{2 R_{0}}\left(-c_{f, \delta} \bar{n}_{f, \delta}+\frac{R_{0}}{r} \bar{n}_{f, \delta^{2}}\right) \tilde{V}_{E} \\
& i \omega \tilde{n}_{f, \delta}^{m=0}=\left(\nabla \cdot n \widetilde{\boldsymbol{V}_{f \perp \mathrm{mag}, \delta}}\right)^{m=0}
\end{aligned}
$$

and

$$
\begin{aligned}
i\left(\omega \mp \omega_{b}\right) \tilde{n}_{f, \delta^{2}}^{m= \pm 1} & =\left(\nabla \cdot n \widetilde{\boldsymbol{V}_{f \perp \mathrm{mag}, \delta^{2}}}\right)^{m= \pm 1} \pm i k\left[\bar{n}_{f, \delta^{0}} \tilde{V}_{f \|, \delta^{2}}^{m= \pm 1}\right. \\
& +\frac{1}{2} \bar{n}_{f, \delta}\left(\tilde{V}_{f \|, \delta}^{m=0}+\tilde{V}_{f \|, \delta}^{m= \pm 2}\right) \\
& \left.+\frac{1}{2} \bar{n}_{f, \delta^{2}}\left(\tilde{V}_{f \|, \delta^{0}}^{m= \pm 1}+\frac{1}{2} \tilde{V}_{f \|, \delta^{0}}^{m=\mp 1}\right)\right] \\
& \pm \frac{i}{8 R_{0}}\left[-\bar{n}_{f, \delta^{2}}\left(1+3 c_{f, \delta^{2}}\right)+3 \frac{R_{0}}{r} \bar{n}_{f, \delta^{3}}\right] \tilde{V}_{E} .
\end{aligned}
$$




\section{Appendix C. Auxiliary equations}

$$
\begin{aligned}
& F_{3}=-\frac{3}{8} \frac{\omega_{b}^{5} q^{2}}{\left(\omega^{2}-\omega_{b}^{2}\right)\left(\omega^{2}-4 \omega_{b}^{2}\right)}, \\
& F_{4}=-\frac{3}{8} \omega_{b}^{5} q^{2} \frac{\partial}{\partial \omega_{b}}\left[\frac{\omega_{b}}{\left(\omega^{2}-4 \omega_{b}^{2}\right)\left(\omega^{2}-\omega_{b}^{2}\right)}\right] \text {, } \\
& B_{3}=\left(1+c_{f}\right)^{3}\left(4-2 c_{f, \delta}+2 \frac{\bar{n}_{f, \delta^{2}}}{\bar{n}_{f, \delta}} \frac{R_{0}}{r}\right) \text {, } \\
& B_{4}=\left(1+c_{f}\right)^{4} \text {, } \\
& F_{5}=-\left[\frac{\omega_{b}^{3} q^{2}}{\omega^{2}-\omega_{b}^{2}}\left(1-3 c_{f, \delta^{2}}+3 \frac{\bar{n}_{\delta^{3}}}{\bar{n}_{\delta^{2}}} \frac{R_{0}}{r}\right)+\frac{2 \omega_{b}^{5} q^{2}}{\left(\omega^{2}-\omega_{b}^{2}\right)^{2}}\left(1+c_{f}\right)\right] \\
& \times \frac{1}{16}\left(1+c_{f}\right)^{3} r \frac{d}{d r} \frac{1}{r} \frac{\bar{n}_{\delta^{0}}^{\prime}}{\omega_{b}} \\
& +\frac{1}{16} \bar{n}_{\delta^{0}} B_{1} \frac{\omega_{b}^{4} q^{2}}{\omega}\left(\frac{1}{\omega-\omega_{b}} \frac{1}{r} \frac{d}{d r} r \frac{1}{\omega-2 \omega_{b}} r^{2} \frac{d}{d r} \frac{1}{r^{2}} \frac{1}{\omega-\omega_{b}}\right. \\
& \left.+\frac{1}{\omega+\omega_{b}} \frac{1}{r} \frac{d}{d r} r \frac{1}{\omega+2 \omega_{b}} r^{2} \frac{d}{d r} \frac{1}{r^{2}} \frac{1}{\omega+\omega_{b}}+\frac{4 \omega}{\omega^{2}-\omega_{b}^{2}} r \frac{d}{d r} \frac{1}{r} \frac{d}{d r} \frac{1}{\omega^{2}-\omega_{b}^{2}}\right) \\
& +\frac{1}{16} \bar{n}_{\delta^{0}} B_{2} \frac{\omega_{b}^{4} q^{2}}{\omega}\left[\frac{1}{\omega-\omega_{b}} \frac{1}{r} \frac{d}{d r} r \frac{1}{\omega-2 \omega_{b}} r^{2} \frac{d}{d r} \frac{1}{r^{2}} \frac{\omega_{b}}{\left(\omega-\omega_{b}\right)^{2}}\right. \\
& +\frac{\omega_{b}}{\left(\omega-\omega_{b}\right)^{2}} \frac{1}{r} \frac{d}{d r} r \frac{1}{\omega-2 \omega_{b}} r^{2} \frac{d}{d r} \frac{1}{r^{2}} \frac{1}{\omega-\omega_{b}} \\
& +\frac{1}{\omega-\omega_{b}} \frac{1}{r} \frac{d}{d r} r \frac{2 \omega_{b}}{\left(\omega-2 \omega_{b}\right)^{2}} r^{2} \frac{d}{d r} \frac{1}{r^{2}} \frac{1}{\omega-\omega_{b}} \\
& -\frac{1}{\omega+\omega_{b}} \frac{1}{r} \frac{d}{d r} r \frac{1}{\omega+2 \omega_{b}} r^{2} \frac{d}{d r} \frac{1}{r^{2}} \frac{\omega_{b}}{\left(\omega+\omega_{b}\right)^{2}} \\
& -\frac{\omega_{b}}{\left(\omega+\omega_{b}\right)^{2}} \frac{1}{r} \frac{d}{d r} r \frac{1}{\omega+2 \omega_{b}} r^{2} \frac{d}{d r} \frac{1}{r^{2}} \frac{1}{\omega+\omega_{b}} \\
& -\frac{1}{\omega+\omega_{b}} \frac{1}{r} \frac{d}{d r} r \frac{2 \omega_{b}}{\left(\omega+2 \omega_{b}\right)^{2}} r^{2} \frac{d}{d r} \frac{1}{r^{2}} \frac{1}{\omega+\omega_{b}} \\
& \left.+\frac{8 \omega \omega_{b}^{2}}{\left(\omega^{2}-\omega_{b}^{2}\right)^{2}} r \frac{d}{d r} \frac{1}{r} \frac{d}{d r} \frac{1}{\omega^{2}-\omega_{b}^{2}}+\frac{8 \omega}{\omega^{2}-\omega_{b}^{2}} r \frac{d}{d r} \frac{1}{r} \frac{d}{d r} \frac{8 \omega_{b}^{2}}{\left(\omega^{2}-\omega_{b}^{2}\right)^{2}}\right] \\
& -\frac{1}{16} \bar{n}_{\delta^{0}}^{\prime} \frac{\omega_{b}^{4} q^{2}}{\omega}\left(\frac{B_{3}}{\omega-\omega_{b}} \frac{d}{d r} \frac{1}{\omega-2 \omega_{b}}+\frac{B_{3}}{\omega+\omega_{b}} \frac{d}{d r} \frac{1}{\omega+2 \omega_{b}}\right. \\
& +B_{4} \frac{\omega_{b}}{\left(\omega-\omega_{b}\right)^{2}} \frac{d}{d r} \frac{1}{\omega-2 \omega_{b}}+B_{4} \frac{1}{\omega-\omega_{b}} \frac{d}{d r} \frac{2 \omega_{b}}{\left(\omega-2 \omega_{b}\right)^{2}} \\
& \left.-B_{4} \frac{\omega_{b}}{\left(\omega+\omega_{b}\right)^{2}} \frac{d}{d r} \frac{1}{\omega+2 \omega_{b}}-B_{4} \frac{1}{\omega+\omega_{b}} \frac{d}{d r} \frac{2 \omega_{b}}{\left(\omega+2 \omega_{b}\right)^{2}}\right] \text {. }
\end{aligned}
$$

\section{References}

[1] R. Nazikian, et al. 101, 185001 (2008).

[2] T. Ido, et al., Nucl. Fusion 51, 073046 (2011). 
[3] W. Chen, et al., Nucl. Fusion 53, 113010 (2013).

[4] N. Winsor, J. L. Johnson, J. M. Dawson, Phys. Fluids 11, 2448 (1968).

[5] R. Fisher, et al., Nucl. Fusion 52, 123015 (2012).

[6] D. Zarzoso, et al., Phys. Rev. Lett. 110, 1 (2013).

[7] G. Fu, Phys. Rev. Lett. 101, 185002 (2008).

[8] Z. Qiu, F. Zonca, L. Chen, Plasma Phys. Control. Fusion 52, 095003 (2010).

[9] Y. I. Kolesnichenko, B. S. Lepiavko, V. V. Lutsenko, Plasma Phys. Control. Fusion 55, 125007 (2013).

[10] L. Wang, J. Q. Dong, Z. He, H. He, Y. Shen, Phys. Plasmas 21, 072511 (2014).

[11] D. Zarzoso, X. Garbet, Y. Sarazin, R. Dumont, V. Grandgirard, Phys. Plasmas 19, 022102 (2012).

[12] H. Wang, Y. Todo, Phys. Plasmas 20, 0 (2013).

[13] H. Wang, Y. Todo, C. C. Kim 110, 155006 (2013).

[14] D. Zarzoso, et al., Nucl. Fusion 54, 103006 (2014).

[15] Z. Qiu, F. Zonca, L. Chen, Phys. Plasmas 19, 082507 (2012).

[16] J. Cao, Z. Qiu, F. Zonca, Phys. Plasmas 22 (2015).

[17] H. Berk, T. Zhou, Nucl. Fusion 50, 035007 (2010).

[18] Z. S. Qu, M. J. Hole, M. Fitzgerald, Phys. Rev. Lett. 116, 095004 (2016).

[19] T. Zhou, MHD GAMs and Kinetic GAMs Driven by Energetic Particles, Ph.D. thesis, University of Texas at Austin (2009).

[20] G. F. Chew, M. L. Goldberger, F. E. Low, Proc. R. Soc. Lond. A. Math. Phys. Sci. 236, 112 (1956).

[21] R. Sgalla, a. Smolyakov, a. Elfimov, M. Bashir, Phys. Lett. A 377, 303 (2013).

[22] R. K. Chhajlani, S. C. Bhand, Journal of Plasma Physics 23, 205 (1980).

[23] M. Fitzgerald, L. Appel, M. Hole, Nucl. Fusion 53, 113040 (2013).

[24] R. Iacono, A. Bondeson, F. Troyon, R. Gruber, Phys. Fluids B 2, 1794 (1990).

[25] Z. S. Qu, M. Fitzgerald, M. J. Hole, Plasma Phys. Control. Fusion 56, 075007 (2014).

[26] H. Sugama, T.-H. Watanabe, Journal of Plasma Physics 72, 825 (2006).

[27] Z. Qiu, L. Chen, Plasma Sci. Technol. 13, 257 (2011).

[28] R. Nazikian (http://w3fusion.ph.utexas.edu/ifs/iaeaep/talks/s1-o2-nazikian-raffi-ep-talk.pdf, 2011).

[29] J.-P. Berenger, Journal of Computational Physics 114, 185 (1994).

[30] P. Lauber, S. Günter, A. Könies, S. D. Pinches, Journal of Computational Physics 226, 447 (2007).

[31] L. Horváth, et al., Nucl. Fusion 56, 112003 (2016). 